Sammlung Metzler

Band 263 


\section{Bernhard Sowinski}

\section{Stilistik}

Stiltheorien und Stilanalysen

J. B. Metzlersche Verlagsbuchhandlung Stuttgart 
Die Deutsche Bibliothek - CIP-Einheitsaufnahme Sowinski, Bernhard:

Stilistik: Stiltheorien und Stilanalysen

Bernhard Sowinski. -

Stuttgart: Metzler, 1991

(Sammlung Metzler; Bd. 263)

ISBN 978-3-476-10263-8

NE: GT

\author{
ISSN 0058-3667 \\ ISBN 978-3-476-10263-8 \\ ISBN 978-3-476-03964-4 (eBook) \\ DOI 10.1007/978-3-476-03964-4
}

\title{
SM 263
}

Dieses Werk einschließlich aller seiner Teile ist urheberrechtlich geschützt. Jede Verwertung außerhalb der engen Grenzen des Urheberrechtsgesetzes ist ohne Zustimmung des Verlages unzulässig und strafbar. Das gilt insbesondere für Vervielfältigungen, Übersetzungen, Mikroverfilmungen und die Einspeicherung und Verarbeitung in elektronischen Systemen.

(C) 1991 Springer-Verlag GmbH Deutschland Ursprünglich erschienen bei J. B. Metzlersche Verlagsbuchhandlung und Carl Ernst Poeschel Verlag GmbH in Stuttgart 1991 


\section{Inhalt}

Vorwort $\ldots \ldots \ldots \ldots \ldots \ldots \ldots \ldots \ldots \ldots \ldots \ldots \ldots, \quad$ IX

Abkürzungen $\ldots \ldots \ldots \ldots \ldots \ldots \ldots \ldots \ldots \ldots \ldots \ldots \ldots \ldots \ldots$ X

1.0 Dimensionen des Stilbegriffs und der Stilistik 1

1.1 Komplexität des Stilbegriffs und der Stilistik ..... 1

1.2 Stil als Kennzeichnungs- und Wertbegriff ...... 3

1.3 Redestil, Sprachstil, Literaturstil, Denkstil ...... 4

1.4 Stilistik, Stilkunde, Stillehre $\ldots \ldots \ldots \ldots \ldots \ldots .6$

2.0 Stilistik und Nachbardisziplinen ............ 8

2.1 Stilistik und Sprachwissenschaft $\ldots \ldots \ldots \ldots \ldots . \ldots$

2.2 Stilistik und Textlinguistik $\ldots \ldots \ldots \ldots \ldots \ldots . . \ldots . \ldots$

2.3 Stilistik und Literaturwissenschaft $\ldots \ldots \ldots \ldots \ldots . \quad 10$

2.4 Stilistik und Sprachdidaktik $\ldots \ldots \ldots \ldots \ldots \ldots \ldots . \ldots \ldots$

2.5 Stilistik und Rhetorik ...................... 14

2.6 Stilistik und Stilkritik $\ldots \ldots \ldots \ldots \ldots \ldots \ldots \ldots . \ldots \ldots$

3.0 Entwicklung des Stilbegriffs und der Stilistik 17

3.1 Der Stilbegriff in der antiken und mittelalterlichen Rhetorik ............................ 17

3.2 Der Stilbegriff in der frühen Neuzeit (15.-18. Jh.) 19

3.3 Stilbegriffe und Stilistik im späten 18. Jh. und im 19. Jh. ........................ 22

3.4 Stilistik und Stilbegriffe im frühen 20. Jh. ...... 26

3.5 Kunstgeschichtlicher Einfluß und geistesgeschichtliche Ausrichtung ................ $\quad 28$

3.6 Der Einfluß der Romanistik in der deutschen Stilistik .............................. 29

3.7 Stilauffassung der >werkimmanenten Interpretation $\ldots \ldots \ldots \ldots \ldots \ldots \ldots \ldots \ldots, \quad 30$

3.8 Linguistische Stilistik: $\ldots \ldots \ldots \ldots \ldots \ldots \ldots \ldots . \ldots \ldots$

Stilstatistik ............................. 32

3.9 Funktionalstilistik ..................... 33

3.10 Strukturalistische Stilistik: ............... 34

Stil als Ergebnis selektiv kombinierter Äquivalenzrelationen - Stil als Resultat der Wahl synonymer SprachmittelStil als Kontrast im Kontext -Stil als Normabweichung 
3.11 Generative Stilistik $\ldots \ldots \ldots \ldots \ldots \ldots \ldots \ldots . \ldots \ldots$

3.12 Stil und Konnotationen ................... 41

3.13 Kommunikative Stilistik $\ldots \ldots \ldots \ldots \ldots \ldots ., 43$

3.14 Pragmatische Stilistik .................... 46

3.15 Gesprächsstilistik $\ldots \ldots \ldots \ldots \ldots \ldots \ldots \ldots . . \ldots$

4.0 Systematische Gruppierung der Stiltheorien $\quad 52$

4.1 Theorien der Stilerlernung $\ldots \ldots \ldots \ldots \ldots \ldots . . \ldots \ldots$

4.2 Theorien der Stilentstehung $\ldots \ldots \ldots \ldots \ldots \ldots . . \ldots 5$

4.3 Phänomenologische Stiltheorien ............. 55

4.4 Kommunikative und pragmatische Stiltheorien .. 56

5.0 Zur Situation der Stilistik in Deutschland und in anderen Ländern ................. 58

5.1 Die heutige Situation der Stilistik in Deutschland . $\quad 58$

5.2 Stilistik in der früheren DDR ............... $\quad 60$

5.3 Romanistische Stilistik .................... 64

5.4 Lateinische Stilistik $\ldots \ldots \ldots \ldots \ldots \ldots \ldots \ldots \ldots \ldots$

5.5 Englische Stilistik $\ldots \ldots \ldots \ldots \ldots \ldots \ldots \ldots \ldots$

5.6 Stilistik in den USA $\ldots \ldots \ldots \ldots \ldots \ldots \ldots \ldots \ldots$

5.7 Skandinavische Stilistik $\ldots \ldots \ldots \ldots \ldots \ldots \ldots . \ldots 6$

5.8 Sowjetische Stilistik $\ldots \ldots \ldots \ldots \ldots \ldots \ldots \ldots . \ldots 6$

6.0 Stilanalyse: Voraussetzungen und Elemente . 71

6.1 Stilauffassungen und Stilanalysen $\ldots \ldots \ldots \ldots \ldots .71$

6.2 Makrostilistik und Mikrostilistik ............. $\quad 72$

6.3 Stilelemente in der Literatur und in Gebrauchstexten ...................... $\quad 74$

6.4 Makrostilistische Einheiten (Elemente): ........ $\quad 75$ Kommunikationsweisen - Stiltypen (Stilarten)/Stilklassen - Funktionalstile - Gruppenstile - Individualstile Epochenstile/Zeitstile - Stilprinzipien - Darstellungsprinzipien - Stilistische Operationen/Stilmuster - Stilzüge - Stilfärbung - Textsorten und Gattungen - Komposition und Bauformen - Darstellungsarten - Redewiedergaben - Erzählstrukturen - Erzählweisen - Erzählsituationen/Erzählverhalten - Erzählhaltung/Darstellungshaltung - Erzählperspektiven - Stilisierung - Parodie/Travestie - Interpretation - Zusammenfassung

6.5 Mikrostilistik: Satzstilistik:

Satzlänge (Kurze Sätze, Sätze mittlerer Länge, Lange Sätze) - Satzgefüge-Satzreduktionen (Aposiopese, Ellipse) - Unterbrechungen der Satzkonstruktion (Prolepse, 
Anakoluth, Bildbrüche/Katachrese, Appositionen, Parenthese, Nachtrag/Ausgliederung) - Wortstellung als Stilmittel-Satzklammer und Ausklammerung - Satzarten als Stilmittel - Paraphrasen - Grammatische Kategorien als Stilmittel

6.6 Übersicht über die rhetorischen Figuren:

Figuren der Hinzufügung (1. Wiederholungsfiguren, 2. Figuren der Häufung), Figuren der Auslassung, Figuren der Stellung und Umstellung.

6.7 Stilmittel des Wortschatzes:

Wortbildungen als Stilmittel - Stilwerte der Wortarten: Substantive, Adjektive, Verben - Verbaler und nominaler Stil - Stilwerte anderer Wortarten (Adverbien, Artikel, Personal- und Possesivpronomen)

6.8 Interpunktion und Typografie als Stilmittel .....

6.9 Lexikalische Stilmittel:

Synonyme - Homonyme/Polysemie - Antonyme Wortfamilien - Wortfelder - Konkreter und abstrakter Wortschatz - Geläufigere und seltenere Wörter - >Normalwortschatz< und Fachwortschatz - >Neutraler< und ideologisch fixierter Wortschatz - Einheimische und fremde Wörter (Fremdwörter) - Überregionaler und regionaler/lokaler Wortschatz - Wörter mit zeitlich begrenzter Geltung (Neologismen, Archaismen, Modewörter, Anachronismen) - Stilschichten im Wortschatz Stilfärbungen

6.10 Bildlichkeit im Text:

Unmittelbare (direkte) Bilder - Mittelbare (indirekte) Bilder - Vergleiche - Tropen: Grenzverschiebungstropen (Umschreibungen, Synekdoche, Emphase, Litotes, Hyperbolie, Metonymie), Sprungtropen (Metapher, Ironie, Allegorie, Personifizierung)

\subsection{Laut- und Klangstilistik}

7.1 Stilrelevante Klangwirkungen ................

7.2 Der Reim und seine stilistische Bedeutung ......

7.3 Metrum und Rhythmus als Stilelemente .........

8.1 Bisherige Vorschläge zur Stilanalyse:

L. Spitzer-J. Petersen - W. Kayser-E. Staiger-M. Riffaterre - G. Michel-H. Graubner-R. Liwerski-E. Riesel - B. Spillner-B. Asmuth-H. Seidler-J. H. Petersen-H. Viebrock - W. Sanders - R. Zimmer - U. Püschel-Autoren i. Spillner (Hg.) $1984-$ B. Sandig - B. ThormannSekulski-Zusammenfassung 
8.2 Die Stilanalyse älterer Texte $\ldots \ldots \ldots \ldots \ldots \ldots . . \ldots \ldots$

8.3 Auswahl-oder Gesamttextanalyse ............ 174

8.4 Stilanalyse und literarische Textinterpretation ... 175

8.5 Stilanalyse, Stilkritik und literarische Wertung .. 179

8.6 Schlußbemerkungen $\ldots \ldots \ldots \ldots \ldots \ldots \ldots \ldots . \ldots 181$

Literaturverzeichnis $\ldots \ldots \ldots \ldots \ldots \ldots \ldots \ldots . \ldots 182$

Stilanalysen zur Literaturgeschichte (Auswahl) $\ldots \ldots \ldots \ldots . \quad 182$

Literatur zur Stilistik (allgemein) ….............. 207

Begriffsregister ............................ 239

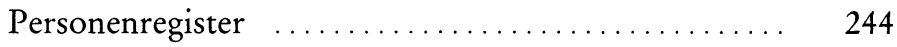




\section{Vorwort}

Die Stilistik gehört zu den Teildisziplinen der Germanistik wie auch jeder anderen Philologie, deren Begriffe oft erwähnt werden, die aber nur wenig systematisch betrieben wird. Neben anderen Gründen ist es oft ein Mangel an umfassend informierender stilistischer Literatur, der solche Defizite bewirkt. Die vorliegende Darstellung versucht, in der Form eines Überblicks über die Entwicklung und die Methoden der Stilistik und ihre Einzelheiten diese Informationslücke zu schließen.

Den Zielen der 'Sammlung Metzler< entsprechend wendet sich das Buch vorwiegend an Germanisten und rückt dementsprechend die deutsche Stilistik in den Vordergrund; der zunehmende internationale Wissensaustausch, vor allem in den linguistischen Disziplinen, ließ es sinnvoll erscheinen, außerdeutsche Entwicklungen der Stilistik und ihre Forschungsliteratur zumindest in Auswahl in unsere Darstellung und unsere Literaturverzeichnisse einzubeziehen. Der Übersichtscharakter des Buches bedingte es, daß Vorzüge und Nachteile verschiedener Stiltheorien und Analysemethoden vorgeführt und verglichen wurden, ohne daß eine Festlegung auf eine bestimmte Richtung erfolgte. Wer für eine bestimmte Stiluntersuchung einen geeigneten Analyseansatz sucht, wird jedoch genügend Literaturhinweise finden.

Obwohl die Literaturlisten keine Vollständigkeit anstreben, sind sie dennoch sehr reichhaltig ausgefallen. Auf diese Weise sollte zumindest ein gewisser Ersatz für eine in der Germanistik noch immer fehlende Stilistik-Bibliographie geboten werden.

$\mathrm{Da}$ das Buch als einführende Übersicht vor allem für Studenten konzipiert ist, versucht es, die verwirrende Fülle der vielen Ansätze zu Stiltheorie und Stilanalyse zu sichten, historisch wie systematisch zu ordnen und exemplarisch und kritisch zu erläutern. Auf ausführliche Diskussionen der einzelnen Richtungen und Ansätze mußte jedoch verzichtet werden.

Köln, im Dezember 1990

B. S. 


\section{Abkürzungen}

$\begin{array}{ll}\text { ABnG } & \text { Amsterdamer Beiträge zur neueren Germanistik } \\ \text { Abdr. } & \text { Abdruck } \\ \text { AnnAcScFennicae. } & \text { Annales Academiae Scientiae Fennicae } \\ \text { Anz } & \text { Anzeiger } \\ \text { ArchfdgesPsych } & \text { Archiv für die gesamte Psychologie } \\ \text { Archiv } & \text { Archiv für das Studium der neueren Sprachen } \\ & \text { und Literaturen } \\ \text { BEDS } & \text { Beiträge zur Erforschung der deutschen Sprache } \\ \text { Beitr } & \text { Beiträge zur Geschichte der deutschen Sprache } \\ & \text { und Literatur (H=Halle/S.; T=Tübingen) } \\ \text { Beitr. rom. Phil. } & \text { Beiträge zur romanischen Philologie } \\ \text { DA od. Diss. Abstr. } & \text { Dissertation Abstracts } \\ \text { DaF } & \text { Deutsch als Fremdsprache } \\ \text { ds } & \text { deutsche sprache } \\ \text { Dt. od.dtsch. } & \text { deutsch(..) } \\ \text { dtv } & \text { deutscher taschenbuch-verlag } \\ \text { DVjs } & \text { Deutsche Vierteljahrsschrift für Literatur und } \\ & \text { Geistesgeschichte } \\ \text { DU } & \text { Der Deutschunterricht } \\ \text { DtUnt } & \text { Deutschunterricht } \\ \text { DuV } & \text { Dichtung und Volkstum } \\ \text { eds } & \text { editors } \\ \text { es } & \text { edition suhrkamp } \\ \text { euphor } & \text { euphorion } \\ \text { EuropHSchrn } & \text { Europäische Hochschulschriften } \\ \text { FAT } & \text { Fischer-Athenäum-Taschenbücher } \\ \text { Festschr. } & \text { Festschrift } \\ \text { FL od FolLing } & \text { Folia Linguistica } \\ \text { ForschSprL } & \text { Forschungen zur Sprache und Literatur } \\ \text { FuF } & \text { Forschungen und Fortschritte } \\ \text { GAG } & \text { Göppinger Arbeiten zur Germanistik } \\ \text { GAL } & \text { Gesellschaft für angewandte Linguistik } \\ \text { GermAbh. } & \text { Germanistische Abhandlungen } \\ \text { GermL } & \text { Germanistische Linguistik } \\ \text { GermRev } & \text { Germanic Review } \\ \text { GLL } & \text { German Life \& Letters } \\ \text { GLS } & \text { Langs Germanistische Lehrbuch-Sammlung } \\ \text { GRM } & \text { Germanisch-Romanische Monatsschrift } \\ \text { GSR } & \text { Gesellschaftswissenschaftlich-Sprachwissen- } \\ & \text { schaftliche Reihe } \\ & \\ & \end{array}$




\begin{tabular}{|c|c|}
\hline H. & Heft \\
\hline $\mathrm{Hg}$. & Herausgeber \\
\hline i. & in \\
\hline InternGermKongr. & Internationaler Germanistenkongreß \\
\hline Jb. & Jahrbuch \\
\hline JIG & Jahrbuch für Internationale Germanistik \\
\hline Kl. Schr.n & Kleinere Schriften \\
\hline $\mathrm{KNf}$ & Kwartalnik neofilologia \\
\hline LGL & Lexikon Germanistische Linguistik \\
\hline $\mathrm{LiLi}$ & Literaturwissenschaft und Linguistik \\
\hline LingArb od. LA & Linguistische Arbeiten \\
\hline LingArbBerKMU & $\begin{array}{l}\text { Linguistische Arbeitsberichte Karl-Marx- } \\
\text { Universität }\end{array}$ \\
\hline Lit. & Literatur; Literary \\
\hline LJb & Literaturwissenschaftliches Jahrbuch \\
\hline Ls/ZISW/A & $\begin{array}{l}\text { Linguistische Studien/Zentral-Institut für Sprach- } \\
\text { wissenschaften der Akademie der Wissenschaften } \\
\text { Berlin/Arbeitsberichte }\end{array}$ \\
\hline LuD & Linguistik und Didaktik \\
\hline M-Diss. & Maschinenschriftliche Dissertation \\
\hline MedAevPhSt & Medium Aevum Philologische Studien \\
\hline MLN & Modern Language Notes \\
\hline MLR & Modern Language Review \\
\hline MLU & Martin-Luther-Universität(Halle/S.) \\
\hline MTU & $\begin{array}{l}\text { Münchner Texte und Untersuchungen zur deut- } \\
\text { schen Literatur des Mittelalters }\end{array}$ \\
\hline Mutterspr & Muttersprache \\
\hline Neophil & Neophilologus \\
\hline NF & Neue Folge \\
\hline NL & Nibelungenlied \\
\hline NS & Die neueren Sprachen \\
\hline PhilPrag & Philologia Pragensia \\
\hline PhilHistKl & Philosophisch-Historische Klasse \\
\hline PhilSchrn & Philologische Schriften \\
\hline PhStuQu & Philologische Studien und Quellen \\
\hline Progr & (Schul)Programm \\
\hline $\mathrm{R}$. & Reihe \\
\hline Respubl. litt. & Respublica litterarum \\
\hline RomForschgn. & Romanistische Forschungen \\
\hline RSiegenBeitr & Reihe Siegener Beiträge \\
\hline SB Ak & Sitzungsberichte Akademie \\
\hline SBd & Sonderband \\
\hline Schrn & Schriften \\
\hline Sekt & Sektion \\
\hline $\mathrm{SH}$ & Sonderheft \\
\hline Slg & Sammlung \\
\hline
\end{tabular}


Spr

SprdGegw

SprKunst

SprPfl

StGen

StuttgArbzGerm

stw

STZ

SuLWU

T.

Teildr.

Unters.

UTB

Vortr.

WB

WdF

Wiss. Beitr.

WW

WZPHE/M

WZHUB

WZUG

WZUH

WZUL

WZUR

Yearb.

ZDL

ZDU

ZfÄsth

ZfdA

ZfdB

ZfDk

$\mathrm{ZfdPh}$

ZfdU

ZfG

ZGL

ZMF

ZPSK

Zs

ZsfangewPsych

ZsfFrzSprLit

ZsfSlaw
Sprache

Sprache der Gegenwart

Sprachkunst

Sprachpflege

studium generale

Stuttgarter Arbeiten zur Germanistik

suhrkamp texte wissenschaft

Sprache im technischen Zeitalter

Sprache und Literatur in Wissenschaft und

Unterricht

Teil

Teildruck

Untersuchungen

Universitäts-Taschenbücher

Vorträge

Weimarer Beiträge

Wege der Forschung (Wiss. Buchgesellsch.

Darmstadt)

Wissenschaftliche Beiträge

Wirkendes Wort

Wissensch. Zeitschr. der Pädagogischen Hochschule Erfurt/Mühlhausen

Wissensch. Zeitschr. der Humboldt-Universität Berlin

Wissensch. Zeitschr. der Universität Greifswald

Wissensch. Zeitschr. der Universität Halle/S.

Wissensch. Zeitschr. der Universität Leipzig

Wissensch. Zeitschr. der Universität Rostock

Yearbook

Zeitschrift Dialektologie und Linguistik

Zeitschrift für Deutschunterricht

Zeitschrift für Ästhetik

Zeitschrift für deutsches Altertum

Zeitschrift für deutsche Bildung

Zeitschrift für Deutschkunde

Zeitschrift für deutsche Philologie

Zeitschrift für deutschen Unterricht

Zeitschrift für Germanistik

Zeitschrift Germanistische Linguistik

Zeitschrift für Mundartforschung

Zeitschrift für Phonetik, Sprachwissenschaft und

Kommunikationsforschung

Zeitschrift

Zeitschrift für angewandte Psychologie

Zeitschrift für Französische Sprache u. Literatur

Zeitschrift für Slawistik 